Bangladesh Journal of Neuroscience 2012; Vol. 28 (1) : 29-37

\title{
Intracranial Aneurysms: Acute VS Delayed Surgery - An Analysis of 52 Cases
}

\author{
SHAMSUL ALAM ${ }^{1}$, ASIFUR RAHMAN $^{1}$, AN WAKIL UDDIN ${ }^{1}$, KM TARIKUL ISLAM $^{1}$, \\ MOSIUR RAHMAN MOJUMDER ${ }^{2}$, MAHFUZUR RAHMAN ${ }^{2}$, ANIS AHMED $^{2}$, ASM ABU OBAIDA $^{2}$, \\ SAIF UL HAQUE ${ }^{2}$, MOHAMMAD NAJIM UDDIN²
}

\begin{abstract}
:
Background: Aneurysm surgery is increasing day by day in our country but the exact timing of surgery is still controversial. Objectives: The aim of this study was to determine the results of early and late surgery for aneurismal subarachnoid haemorrhage. The aim of microneurosurgical management of an aneurysm is the total occlusion of the aneurysm sac by clipping at the neck of aneurysm with preservation of flow in the parent artery and preservation of all its perforating arteries with minimal or no brain retraction. Methods: There were 52 patients included in this study among them 3 patients expired soon after the admission before surgery could take place. Hence 49 patients underwent clip surgery from July 2005 to May 2012 for 52 aneurysms because 3 patients harboured multiple aneurysms. Patient's history, clinical findings, Hunt \& Hess grading, Fisher grading of CT scan, preoperative \& postoperative CT angiography, postoperative outcome were collected and analyzed. Results: Most of the clipping (57.14\%) were done in intermediate stage ( $4^{\text {th }}$ to $10^{\text {th }}$ days), because patients usually referred from peripheral hospital on 2 nd or $3^{\text {rd }}$ day after the acute SAH Those who was admitted early and H\&H status good, was fit to do early surgery (within $3^{\text {rd }}$ day), (28.57\%). Overall outcome was assessed at 3 months after SAH using the Glasgow Outcome Scale. Good outcome were observed in 40 cases among them 22 cases (42.3\%) were able to return premorbid activities. Total mortality in this series were 10 cases $(19.23 \%)$ which includes preoperative death while waiting for clipping -3 cases and postoperative death- 7 cases(14.2\%). Conclusion: There is no reason to postpone clipping surgery in patients who are eligible for surgery at day 5. Surgery after day 10 is associated with worse outcome. Although these studies is having high rate of mortality which can be progressively minimize by our continuous improvement of surgical skills and postoperative critical care management of aneurysm patients.
\end{abstract}

Key word: aneurysm, craniotomy, clipping.

\section{Introduction:}

Aneurysms resemble bubbles or focal dilation of arteries that occur at weak points of the artery wall (figure-1). There are many factors for its formation. These factors include genetic predisposition, the anatomy of the artery and its branches, 'wear-andtear' on the wall of the arteries due to blood flow, artery disease and cigarette smoking ${ }^{1}$. Aneurysms are the most common at circle of Willis in the central skull base. Approximately $80 \%$ of aneurysms arises from anterior circulation of the brain, while 20\% form posterior circulation of the brain ${ }^{1.2}$.

It is uncommon to diagnose an aneurysm before it has ruptured and most people with aneurysms are unaware that they have an aneurysm until it bursts. Overall 3.6-6\% of normal population has aneurysm, among them 1.4-1.9 \% rupture in a year. Women have more tendencies to rupture. There is seasonal variation of rupture ${ }^{3}$.

1. Assistant Professor, Department of Neurosurgery, Bangabandhu Sheikh Mujib Medical University, Dhaka.

2. Resident, Department of Neurosurgery, Bangabandhu Sheikh Mujib Medical University, Dhaka. 
It is known that the majority of aneurysms that have ruptured are less than $10 \mathrm{~mm}$ in size ${ }^{3.4}$. Over the next 5 years after diagnosis an average aneurysm between 2 and $6 \mathrm{~mm}$ in size has a risk of bleeding between 1 and $2 \%$, an aneurysm between 7 and 9 $\mathrm{mm}$ size has a five year risk of bleeding of $6 \%$, an aneurysm between 10 and $24 \mathrm{~mm}$ in size has a five year risk of bleeding of $11 \%$ and larger aneurysms have a $28 \%$ chance of bleeding ${ }^{3.4}$.

The history of intracranial aneurysm surgery is not a long one. The first direct operation on an intracranial aneurysm was performed by Norman Dott, who wrapped a ruptured aneurysm in 1933, and the first clipping of an aneurysm was performed by Walter Dandy in $1938^{5.6}$. The results of surgery improved dramatically when the operating microscope was introduced in the 1960s and a subsequent improvement followed the use of the calcium antagonist nimodipine and the maintenance of a high fluid intake to lessen the risk of delayed cerebral ischemia ${ }^{7}$. For many years clipping for the aneurysm was regarded as the definitive mode of treatment, but the development of the GDC coil in 1990 allowed an alternative approach that avoided the hazards of open surgery ${ }^{7}$.

After an aneurismal subarachnoid haemorrhage, there are two major sources of morbidity and mortality: rebleeding and delayed ischemia secondary to cerebral vasospasm. The incidence of rebleeding is greatest immediately after the initial haemorrhage, and the incidence of vasospasm is highest between the 5th and 9th days after the ictus ${ }^{8}$. It thus seems logical to proceed with early surgery, such an approach is certainly the best means for eliminating rebleeding as a source of morbidity and mortality. Early surgery also facilitates the management of cerebral vasospasm by volume expansion and induced hypertension manoeuvres that are quite risky in the patient with an untreated aneurysm ${ }^{8}$.

The anterior communicating artery (AcomA) is a recognized site of aneurysm predilection accounting for more than one fourth of all cerebral aneurysms in several large studies ${ }^{9}$. Because of

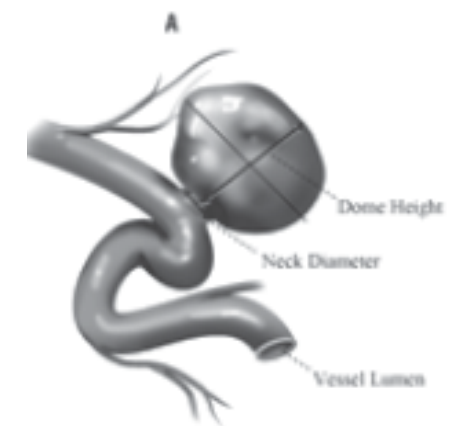

Fig.-1: Showing various dimensions of an aneurysm

the complexity and diversity of the geometry and flow conditions in the AcomA, it is not surprising that aneurysms of the AcomA are considered the most complex of the anterior circulation. It is widely believed that the initiation, growth, and, ultimately rupture of cerebral aneurysms are related to the interaction between hemodynamic forces with the arterial wall biology, resulting in a localized weakening of the wall. Aneurysms of the AcomA complex are more likely to have asymmetric A1 segments and furthermore, to have exclusive filling angiographically from A1 segment in up to $78 \%$ cases. $^{9}$

Posterior communicating artery (PCOM) aneurysms are another most common aneurysms encountered by neurosurgeons and neurointerventional radiologists and are the second most common aneurysms overall ( $25 \%$ of all aneurysms) representing $50 \%$ of all internal carotid artery (ICA) aneurysms ${ }^{10}$. Not only these aneurysms can present with a typical subarachnoid haemorrhage, but also they can present with an isolated oculomotor nerve palsy (OMNP) or a non-traumatic subdural hematoma (SDH).

Jane et al, evaluated the risk of rehaemorrhage in ruptured ACOM and PCOM aneurysm, finding a $50 \%$ risk of rerupture within the initial six months followed by $3.5 \%$ per year thereafter ${ }^{11}$.

The surgical treatment of basilar tip aneurysms remains one of the most difficult tasks in neurosurgery because the view is obscured due to the depth of the aneurysm, overlapping neurovascular and bony structures, and the proximity of perforators ${ }^{12}$. 
Despite the many studies about timing for surgery in subarachnoid haemorrhage (SAH), the optimum time is still in debate. The aim of this study was to determine the results of early and late surgery for aneurysmal subarachnoid haemorrhage. The proponents of early surgery focused on reduction of the devastating effects of aneurysmal re-bleeding within the first 2 weeks and its high mortality. On the other hand, some authors believe that delayed surgery may be better choice, because operating on the acutely injured brain may be associated with high risk for surgical morbidity and mortality ${ }^{13}$.

Some studies advocates surgery neither early nor late, and indicate that the intermediate period in 4 to 10 days after the SAH is a risky time for surgery, because during this period the risk for cerebral vasospasm and ischemia may be very high ${ }^{14}$.

Unlike the authors, it is our belief that early aneurysm surgery is a technically more challenging procedure than delayed surgery. Despite the use of osmotic agents, hyperventilation, and cerebrospinal fluid drainage, it is our opinion that the brain is more friable and difficult to retract safely.

However, Shabepour et al. study in Iran had some results different from most previous studies; they evaluated 110 aneurysmal SAH and reported that the complications of late surgeries were significantly lower than early surgeries; the complication rate in surgeries performed during first 3 days after SAH was $66.7 \%$. This rate was $54.57 \%$ for surgeries in 3 to 14 days after $\mathrm{SAH}$, and $22.9 \%$ for surgeries after 14 th days ${ }^{15}$.

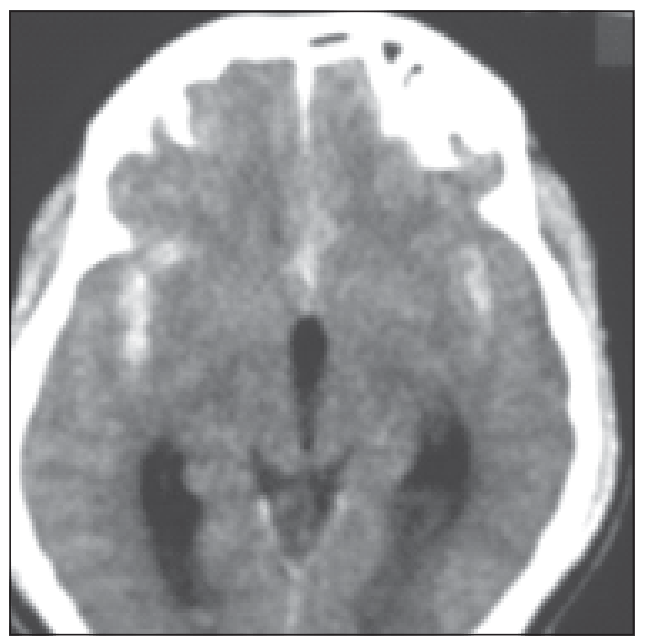

Fig.-2: CT scan shows of SAH, Fisher grade 2.
The aim of microneurosurgical management of an aneurysm is the total occlusion of the aneurysm sac by clipping at the neck of aneurysm with preservation of flow in the parent artery and preservation of all its perforating arteries with minimal or no brain retraction.

\section{Methods:}

There were 52 patients included in this study among them 3 patients expired soon after the admission before surgery could take place. Hence 49 patients underwent clip surgery from July 2005 to May 2012 for 52 aneurysms because 3 patients harbored multiple aneurysms. Patients' history, clinical findings, Hunt \& Hess grading, Fisher grading of CT scan (figure-2), preoperative \& postoperative CT angiography, postoperative outcome were collected and analyzed.

About $90 \%$ of aneurysms were accessible via a standard frontotemporal (pterional) craniotomy centered over the pterion. Only occasional cases, aneurysms of the distal anterior cerebral artery and the lower vertebrobasilar trunk, require different surgical approaches. The risk of intraoperative rupture of the aneurysm, which occurs in 5-10\% of cases, can be minimized by induction of hypotension. This seems a safe measure provided that the anaesthetist ensures maintenance of a high blood volume. We preferred not to apply temporary clips to the main artery proximal to the aneurysm because it was associated with a high incidence of ischemic cerebral damage. Temporary clipping was certainly not tolerated if the blood pressure is lowered at the same time.

\section{Surgical steps}

Acom aneurysm: Pterional craniotomy is commonly done for this. We prefer to approach from right side however sometimes it needed to approach from left side when. Left A1 is dominant and Ipsilateral and contralateral $A 2$ is well visualized from left side. We prefer 5-10 degree rotation to the contralateral side to keep the figure of $\mathrm{H}$ in vertical position. Wide sylvian fissure dissection was done for gentle retraction of frontal lobe. Anterior interhemispheric fissure was dissected to avoid gyrus rectus resection (figure-3A,3B,3C). 


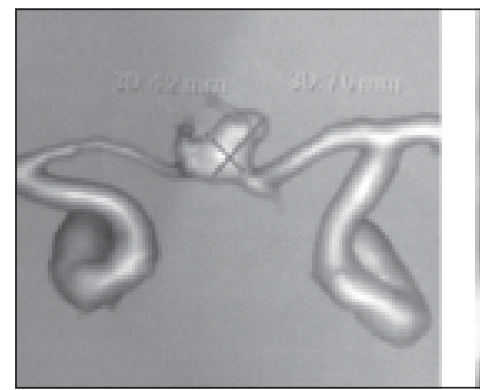

A

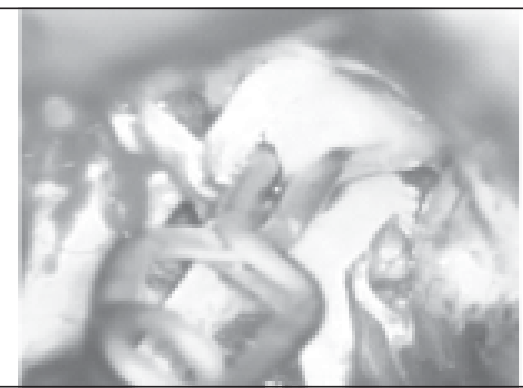

B

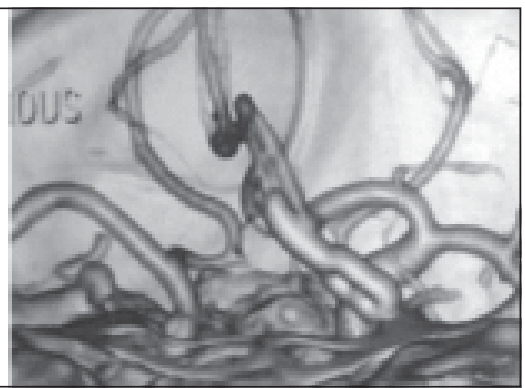

C

Figure-3: CT angiogram shows ACOM aneurysm (A), Peroperative clipping of ACOM aneurysm (B) \& Post clip angiogram shows no residual aneurysm (C).

Pcom aneurysm: Pterional craniotomy with rotation 60-75 degree to contralateral side for posteriorly directing aneurysm which allows the aneurysm to be seen in profile with carotid artery. The oculomotor nerve should be identified and protected. It is not necessary to expose the aneurysm dome. When the inferior and superior aspect of aneurysm has been identified and adjacent arteries made free, then the aneurysm can be safely clipped.

MCA aneurysm: Pterional approach with turning the patient head 45 degrees to contralateral side means the operative pathway will be almost vertically downward along the sphenoid ridge. This reduces the need for retraction of temporal lobe, and often only needs the frontal lobe to be retracted. The head is also extended to allow the frontal lobe to fall. Finally the rotated, extended head is elevated upward to facilitate venous return.

\section{Basilar top aneurysm:}

Pterional craniotomy with head is positioned in a Mayfield head holder with the head elevated above the shoulder level and 20 dergree rotation to the contralateral side away from the operative side. The head is extended until the maxillary eminence is highest point in the field. A pterional craniotomy is then performed followed by drilling of sphenoid ridge and orbital roof until a flat surface is achieved so that straight trajectory to the proximal carotid can be visualized along the skull base.

For preexisting hydrocephalus or in case of brain swelling during surgery we are in need of brain relaxation. This can be done by a ventricular catheter placed in "paine's point". This is done by aiming perpendicularly to the triangle $2.5 \mathrm{~cm}$ back along the sylvian fissure and $2.5 \mathrm{~cm}$ superiorly.

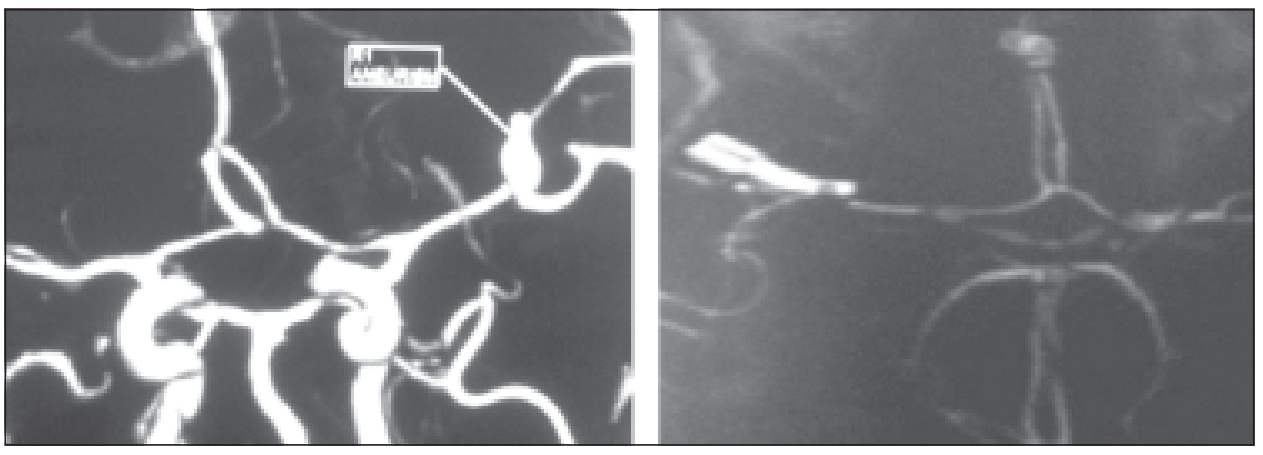

A

B

Figure 4: CT angiogram shows left MCA aneurysm $(A)$ \& Post-clip angiogram shows no residual aneurysm (B). 
Results:

The mean age group of our study was 45.7 years. Age varies from 16- 70 years. Male: female ratio was 1:08. In our study male are commoner then female.

Hunt and Hess grades at admission are summarized in Table I. Thirty seven patients (71.2\%) were classified as Grade I-II, Twelve patients (23.0\%) as Grade III-IV. 3 patients (5.7\%) as Grade V.

Table-I

Distribution of Hunt \& Hess Grading: $(n=52)$

\begin{tabular}{lc}
\hline Hunt \& Hess Grading & No. of cases \\
\hline I - II & $37(71.215 \%)$ \\
III-IV & $12(23.07 \%)$ \\
V & $3(5.76 \%)$ \\
\hline
\end{tabular}

The subarachnoid clot thickness -Fisher grading at admission were summarized in Table-II. 84.4\% of cases were in grade I-II. 4 cases(7.6\%) were in grade III-IV. Only 4 cases (7.6\%) were grade V.

Table-II

Distribution of Fisher grade of Aneurysms: $(n=52)$

\begin{tabular}{lc}
\hline Fisher Grading & No. of cases \\
\hline I - II & $44(84.61 \%)$ \\
III-IV & $4(7.69 \%)$ \\
V & $4(7.69 \%)$ \\
\hline
\end{tabular}

Cerebral CT angiography was performed at admission in all patients (angiography was performed within 48 hours of haemorrhage onset in most of the cases). The location and size of the ruptured aneurysm were obtained from a review of angiographic images. Commonest aneurysm found in ACOM location which was 42.30\%. Next commoner the PCOM which constitute $26.92 \%$ and MCA aneurysms were $9.61 \%$. Basilar top aneurysms were found only in 4 cases $(7.6 \%)$ (Table-III). The aneurysm was single in $92.9 \%$ and multiple aneurysms were found in 3 cases (5.76\%).
Table-III

Distribution of CT angiographic findings of Location of Aneurysms:( $n=52)$.

\begin{tabular}{lc}
\hline Location of Aneurysms & No. of aneurysms \\
\hline ACOM aneurysm & $22(42.30 \%)$ \\
PCOM aneurysm & $14(26.92 \%)$ \\
MCA aneurysm & $5(9.61 \%)$ \\
ICA aneurysm & $5(9.61 \%)$ \\
DACA aneurysm & $2(3.84 \%)$ \\
Basilar top aneurysm & $4(7.69 \%)$ \\
Paraclinoid aneurysm & $2(1.92 \%)$ \\
Vertibrobasilar junctional aneurysm & $1(1.92 \%)$ \\
\hline Total & $52(100 \%)$ \\
\hline
\end{tabular}

Aneurysm size was categorized in three groups. Small sizes were between 4-10 mm - constitute the commonest size which were $84.6 \%$. Large sizes were between $11-25 \mathrm{~mm}$ constitute $11.5 \%$ and $>25 \mathrm{~mm}$ were giant aneurysm which was only2 cases(Table IV).

Table-IV

Distribution of Size of Aneurysms, ( $n=52)$

\begin{tabular}{lc}
\hline Size of Aneurysms & No. of Aneurysms \\
\hline $4 \mathrm{~mm}-10 \mathrm{~mm}$ & $44(84.61 \%)$ \\
$11 \mathrm{~mm}-25 \mathrm{~mm}$ & $6(11.53 \%)$ \\
$<25 \mathrm{~mm}$ & $2(3.84 \%)$ \\
\hline Total & 52 \\
\hline
\end{tabular}

Pterional craniotomy (91.8\%) was the most common approach for most of the aneurysm both for almost all anterior and some posterior circulation aneurysm. Contralateral pterional approach were done in 3 cases(6.12\%). For vertebrobasilar junction retrosigmoid suboccipital craniectomy was choiced. For DACA aneurysm anterior interhemispheric approach was chosed.(Table-V).

Table-V

Distribution of Name of Surgery $(n=49)$

\begin{tabular}{lc}
\hline Name of Surgery & No. of cases \\
\hline Pterional Crainotomy & $45(91.83 \%)$ \\
Orbitopterional Crainotomy & $1(2.04 \%)$ \\
Contralateral Pterional Crainotomy & $3(6.12 \%)$ \\
Retrosigmoid suboccipital approach & $1(2.04 \%)$ \\
\hline Total & $49(100 \%)$ \\
\hline
\end{tabular}

Most of the surgery(57.14\%) were done in intermediate $\operatorname{stage}\left(4^{\text {th }}\right.$ to $10^{\text {th }}$ days), because 
patients usually referred from peripheral hospital on $2^{\text {nd }}$ or $3^{\text {rd }}$ days after the acute SAH. Those who was admitted early and $\mathrm{H} \& \mathrm{H}$ status good, was fit to do early surgery (within $3^{\text {rd }}$ day) (28.57\%). Those who were poor $\mathrm{H} \& \mathrm{H}$ grade at admission and or CT angiogram reveled sign of vasospasm and those having medical co-morbidity such as asthma, coronary ischemia, were not able to do early or intermediate surgery hence they were selected for late surgery(after $11^{\text {th }}$ day onward)(table-VI). Two patients who died from rebleeding while waiting for surgery although they were fit for surgery at anytime. Another one patients died from severe vasospasm so soon after admission that surgery could not have been performed.

\section{Table-VI}

Distribution of Day of Aneurysm Surgery ( $n=49)$

\begin{tabular}{lc}
\hline Day of Aneurysm Surgery & No. of aneurysms \\
\hline $1^{\text {st }}$ day $-3^{\text {rd }}$ day & $14(28.57 \%)$ \\
$4^{\text {th }}-10^{\text {th }}$ day & $28(57.14 \%)$ \\
$>11^{\text {th }}$ day & $7(14.28 \%)$ \\
\hline Total & $49(100 \%)$ \\
\hline
\end{tabular}

Overall outcome was assessed at 3 months after SAH using the Glasgow Outcome Scale. Good outcome were observed in 40 cases among them 22 cases $(42.3 \%)$ were able to return premorbid activities. Poor outcome was defined by the Glasgow Outcome Scale criteria of death, vegetative state, or severe disability. Total mortality in this series were 10 cases (19.23\%) which includes preoperative death while waiting for clipping -3 cases and postoperative death -7 cases( 14.2\%) (Table -VII).

Table-VII

Distribution of Glasgow outcome scale ( $N=52)$

\begin{tabular}{|c|c|}
\hline Good outcome & No. of cases \\
\hline 1. Return to premorbid occupation & $22(42.30 \%)$ \\
\hline $\begin{array}{l}\text { 2. Neurologically normal, not } \\
\text { returned to premorbid occupation }\end{array}$ & $10(19.23 \%)$ \\
\hline 3. Independent, mild neurological deficit & $5(9.61 \%)$ \\
\hline $\begin{array}{l}\text { Poor outcome } \\
\text { 4. Dependent, significant deficit }\end{array}$ & $5(9.61 \%)$ \\
\hline $\begin{array}{l}\text { 5. Dead(preop-3cases }+(14.2 \%) \\
\text { postop-7cases }\end{array}$ & $10(19.23 \%)$ \\
\hline Total & $52(100 \%)$ \\
\hline
\end{tabular}

Commonest complication of aneurysm sugery were rerupture during dissection of aneurysm sac which occurred $7.6 \%$ cases. Incompletely clipped aneurysm also reruptured in postoperative period which occurred $3.8 \%$ cases. Postoperative severe hypotension developed in $5.7 \%$ cases. Most common causes of postoperative mortality in our series were from hypotension and improper management of vasospasm. Rerupture from incompletely clipped aneurysm were accounted 2 cases which lead to death(table-8). Postoperative vasospasm \& limb weakness were another common problem for which we need to manage by ionotrophic agent like dopamine, adrenaline \& dobutamine. Subdural hematoma, meningitis, and acute and late hydrocephalus were some minor complications (Table- VIII).

Table-VIII

Distribution of Complications of Aneurysm ( $n=52)$

\begin{tabular}{lc}
\hline Complication & No. of cases \\
\hline Re-rupture while waiting & $2(3.84 \%)$ \\
Preop severe vasospasm & $1(1.92 \%)$ \\
Intraoperative rupture & $4(7.69 \%)$ \\
Post operative rupture & $2(3.84 \%)$ \\
Post operative hypotension & $3(5.76 \%)$ \\
Pre operative vasospasm & $6(11.53 \%)$ \\
Newly developed Post operative & $5(9.61 \%)$ \\
vasospasm \& hemiplegia & \\
Post operative subdural haematoma & $2(3.84 \%)$ \\
Meningitis & $5(9.61 \%)$ \\
Hydrocepalus & $3(5.76 \%)$ \\
Tension pneumocephalus & $1(1.92 \%)$ \\
VP shunt & $3(5.76 \%)$ \\
No complications & $15(28.84 \%)$ \\
\hline Total & $52(100 \%)$ \\
\hline
\end{tabular}

\section{Discussion:}

Pterional craniotomy was the most common approach for both anterior and posterior circulation aneurysm ${ }^{16}$. However some author choose orbitopterional craniotomy in case of ACOM aneurysm in acute setting. In one study overall outcomes at discharge using the Glasgow outcome scale of those who underwent pterional craniotomy were good in 52 (69.4\%) patients, fair in 13 
(17.3\%), and poor in 10 (13.3\%) among 75 cases of ACOM aneurysm. At last follow-up after 6 months of surgery, outcomes were good in $63(84 \%)$ patients, fair in $6(8 \%)$, and poor in $6(8 \%)$. Disability included mild in $10 \%$, partial in $18.8 \%$, moderate in $8.6 \%$, moderately severe in $1.4 \%$, severe in $2.9 \%$, extremely severe in $2.9 \%$, and vegetative state in $1.4 \%$. Overall $74 \%$ of patients returned to work after 4 months, $83 \%$ of previously unemployed patients returned to baseline, and $25 \%$ were disabled ${ }^{17}$. In this study good outcome were observed in 40 cases among them 22 cases (42.3\%) were able to return premorbid activities. Poor outcome were observed in 15 cases among them 7 cases were died following surgery(14.2\%) Here our postoperative mortality was quite high $(14.2 \%)$, this was probably from interaction of many factors -such as patient factor-delay admission, hesitation regarding giving consent for operation in good $\mathrm{H} \& \mathrm{H}$ status, postoperative poor nursing management, and surgeons skill.

Samson et al. reported that the outcome and complications of early surgery on first 8 days after $\mathrm{SAH}$ were not different from late surgery in 9 to 31days after $\mathrm{SAH}$, but ischemic events after early surgery were significantly higher ${ }^{18}$.

Temporary clipping and projection of the aneurysm did not affect the outcome. Causative factors of unfavorable outcomes were primary brain damage by haemorrhage in cases of small and large aneurysms and perforator damage in the case of giant aneurysm. Poor clinical $\mathrm{H} \& \mathrm{H}$ grade and vasospasm are the causative factors of poor outcome in patients with ruptured aneurysm. The poor outcome could also have been correlated with poor clinical condition at admission, early rebleeding, or early deterioration from other causes $^{19}$.

Once the neck of the aneurysm was adequately exposed, then we must pay significant attention to preservation of the parent artery, perforators without significant manipulation of the fundus ${ }^{19}$. Leipzig et al, reviewed a large series of aneurysm clipping looking for risk factors of intraoperative rupture. PCOM aneurysms had the second highest rate of intra-operative rupture (second only to
ACOM aneurysms) amongst anterior circulation aneurysms ${ }^{20}$.

A strong correlation was found between rehaemorrhage and residual aneurysm. Risk of rehaemorrhage increased from $1.1 \%$ in completely occluded aneurysm to $17.6 \%$ in a partially treated aneurysm where residual filling of the dome was left untreated. Also the median time to rerupture was only three days ${ }^{19}$. In our series 2 cases developed reruptured in early postoperative period among 49 cases of operatively treated aneurysm (table-VIII)

Aneurysm surgery are increasing day by day, probably because of improvement of motivation of the patient party, availability of investigation and availability of aneurysm clip of various size and shape in our country.(Diagram-1)

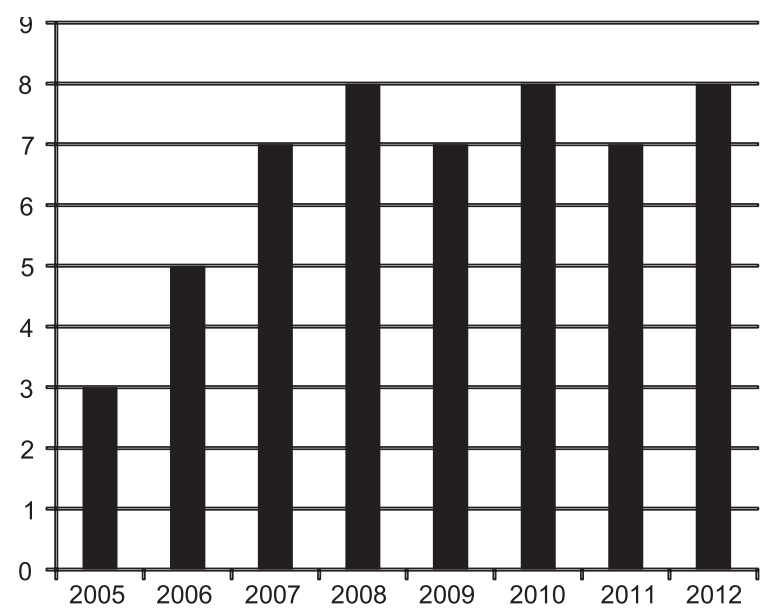

Diagram-I Bar diagram of aneurysm cases in year. $(n=52)$.

There are 3 possible explanations for the observed better outcomes among SAH patients treated within 72 hours of admission: (1) the death and disability associated with rebleeding is reduced; (2) the death and disability related to cerebral vasospasm is reduced because more intensive measures can be undertaken with secured aneurysms; and (3) early treatment is a marker of higher performance on several quality care parameters 20 .

The international cooperative study on the timing of aneurysm surgery recruited 3521 patients with aneurusmal SAH. There was no difference in good 
outcome defined by Glasgow outcome scale at 6 months in early (0-3day) and delayed (11-14 day) surgery group but lower rate were observed in intermediate (7-10 day) surgery group. The rate of rebleeding in early surgery group $5.7 \%$ in compared with $13.9 \%$ delayed surgery group ${ }^{21}$. A subgroup analysis on north American population(772 patients ) demonstrates high rate of good outcome in early vs delayed group (70.9\%$61.7 \%$ ) respectively even though there is no mortality. A recent data reevaluate the definition of early treatment (0-2 days vs $0-3$ days) proposed by international cooperative study trail. A major benefit of early surgery lies in the decreasing frequency of rebleeds that occurs in the interim period before aneurysm treatment is performed ${ }^{22}$.

In our study the hospital stay in early surgery group was significantly lower than late group. This finding is concordant with Ross et al. ${ }^{23}$ and Bolander et al. studies 24 .

\section{Conclusion:}

In conclusion, this study revealed that most of the aneurysm surgery were done between $4^{\text {th }}$ to $10^{\text {th }}$ days of post acute SAH, which reflects that timing of surgery should be individualized for each patient based on clinical situation such as age, $\mathrm{H} \& \mathrm{H}$ Grading, Fisher Grading, size and site of aneurysm, presence or absence of vasospasm and other medical comorbid factors. There is no reason to postpone clipping surgery in patients who are eligible for surgery at day 5 . Surgery after day 10 is associated with worse outcome. Although this study is having high rate of mortality which can be progressively minimize by our continuous improvement of surgical skills and postoperative critical care management of aneurysm patients.

\section{References:}

1. McDougall CG, Spetzler RF, Zabramski JM, Partovi S, Hills NK, Nakaji P, et al. The Barrow Ruptured Aneurysm Trial . J Neurosurg . 2012;116:135-44

2. Juvela S, Porras M, Poussa K. Natural history of unruptured intracranial aneurysms: probability and risk factors for aneurysm rupture. Neurosurg Focus. 2000;8(5)
3. Andaluz N, Zuccarello M. Recent trends in the treatment of cerebral aneurysms: analysis of a nationwide inpatient database. J Neurosurg. 2008;108(6):1163-9.

4. Ishibashi T, Murayama $\mathrm{Y}$, Urashima M, Saguchi T, Ebara M, Arakawa $\mathrm{H}$, et al. Unruptured intracranial aneurysms: incidence of rupture and risk factors. Stroke. 2009;40:313-6

5. Dott NM. Intracranial aneurysms cerebral arterio-radiography and surgical treatment. Edinb Med J 1933;40: 219-34.

6. Dandy WE. Intracranial aneurysm of the internal carotid artery cured by operation. Ann Surg 1938;107: 654-9.

7. Sahs AL. Cooperative study of intracranial aneurysms and subarachnoid hemorrhage. Report on a randomized treatment study. I. Introduction. Stroke. 1974;5(4):550-51.

8. Suzuki J, Onuma T, Yoshimoto T. Results of early operations on cerebral aneurysms. Surg Neurol. 1979;11(6):407-12.

9. Suzuki J, Yoshimoto T. Early operation for the ruptured intracranial aneurysm. Jpn J Surg. 1973;3(3):149-56.

10. Norlen G, Olivecrona $\mathrm{H}$. The treatment of aneurysms of the circle of Willis. J Neurosurg. 1953;10(4):404-15.

11. Jane JA, Kassell NF, Torner JC, Winn HR. The natural history of aneurysms and arteriovenous malformations. J Neurosurg. 1985;62:321-3.

12 Tytus JS, Ward AA Jr. The effect of cervical carotid ligation on giant intracranial aneurysms. J Neurosurg. 1970;33(2):184-90.

13. Kassell NF, Torner JC, Haley EC. The International Cooperative Study on the timing of Aneurysm Surgery. Part 1: overall management results. J Neurosurg 1990;73: 18-36.

14. Mahaney KB, Todd MM, Torner JC. Variation of patient characteristics, management, and outcome with timing of surgery for aneurysmal subarachnoid haemorrhage. J Neurosurg. 2011;114(4):1045-53. 
15. Shabehpoor M, Arjmand A, Safdari $\mathrm{H}$, Azhari Sh,Naebaghaee H, Mohammadi H. Outcome of cerebral aneurysm surgery (early surgeryrelated complication and outcome after aneurysm clip placement). Iran J Surg 2006;14(2).

16. Clatterbuck RE, Tamargo RJ. Contralateral approaches to multiple cerebral aneurysms. Neurosurgery. 2005;57(1 suppl):160-63.

17. Adams CB, Loach AB, O'Laoire SA. Intracranial aneurysms: analysis of results of microneurosurgery. BMJ 1976; 607-9.

18. Samson DS, Hodosh RM, Reid WR, Beyer CW, Clark WK. Risk of intracranial aneurysm surgery in the good grade patient: early versus late operation. Neurosurgery. 1979;5(4): 422-6.

19 Spetzer V, Gilsbach JM. Results of early aneurysm surgery in poor-grade patients. Neurol Res 1994;16: 27-30.

20. Ljunggren B, Brandt L, Sundbarg G, Saveland $\mathrm{H}$, Cronquist $\mathrm{S}$, Stridbeck $\mathrm{H}$. Early management of aneurysmal subarachnoid haemorrhage. Neurosurgery. 1982;11(3): 412-8.

21. Intracranial Subarachnoid Aneurysm Trial (ISAT) Collaborative Group. Intracranial
Subarachnoid Aneurysm Trial (ISAT) of neurosurgical clipping versus endovascular coiling in 2143 patients with ruptured intracranial aneurysms - a randomised trial. Lancet 2002;360: 1267-74.

22. Molyneux AJ, Kerr RS, Yu LM. International subarachnoid aneurysm trial (ISAT) of neurosurgical clipping versus endovascular coiling in 2143 patients with ruptured intracranial aneurysms: a randomised comparison of effects on survival, dependency, seizures, rebleeding, subgroups, and aneurysm occlusion. Lancet. 2005; 366 (9488): 809-17.

20. Leipzig TJ, Redelman K, Horner TG.Reducing the risk of rebleeding before early aneurysm surgery: a possible role for antifibrinolytic therapy. J Neurosurg. 1997;86(2):220-5.

23. Ross $\mathrm{N}$, Hutchinson PJ, Seeley $\mathrm{H}$, Kirkpatrick PJ. Timing of surgery for supratentorial aneurysmal subarachnoid hemorrhage: report of a prospective study. J Neurol Neurosurg Psychiatry. 2002 Apr;72(4):480-4.

24. Bolander HG, Kourtopoulos H, West KA.Retrospective analysis of 162 consecutive cases of ruptured intracranial aneurysms. Total mortality and early surgery. Acta Neurochir (Wien). 1984;70(1-2):31-41. 